\title{
Insight into Goal-Directed Movements: Beyond Fitts' Law
}

\author{
Karin Nieuwenhuizen ${ }^{1}$, Dzmitry Aliakseyeu ${ }^{2}$, and Jean-Bernard Martens ${ }^{1}$ \\ ${ }^{1}$ Eindhoven University of Technology, Department of Industrial Design, Den Dolech 2, \\ 5600 MB Eindhoven, The Netherlands \\ \{C.J.H.Nieuwenhuizen, J.B.O.S.Martens\} @tue.nl \\ ${ }^{2}$ Philips Research, Media Interaction, High Tech Campus 5, \\ 5656 AE Eindhoven, The Netherlands \\ Dzmitry.Aliakseyeu@philips.com
}

\begin{abstract}
Various methods and measures have been developed to assess the quality of input devices and interaction techniques. One approach to investigating the performance of input devices and interaction techniques is to focus on the quality of the produced movements. The current paper proposes a new method of analyzing goal-directed movements by dividing them into meaningful phases. In addition to the proposed analysis method a selection of measures is suggested to assess different aspects of rapidly aimed movements. In order to evaluate the added value of the proposed analysis method an experiment has been conducted to compare two input devices (mouse versus stylus with tablet) with respect to their performance on a multi-directional pointing task. The results show that the analysis into several phases reveals clear differences in the movement strategy.
\end{abstract}

Keywords: Input devices, interaction techniques, movement analysis, performance measures.

\section{Introduction}

A key aspect of human-computer interaction is the use of input devices, such as keyboard, mouse, or tablet with stylus. Since millions of people use computers on a daily basis it is important that input devices are well tailored to users' needs. This is especially true when devices are designed for people with disabilities [9, 11], or when devices are used within challenging environments, such as virtual reality. To improve existing input devices or in the process of developing new ones it is necessary to identify the reasons for variations in performance.

Several studies have compared input devices or interaction techniques by observing characteristics of movement paths during the execution of basic tasks such as pointing, selecting or steering $[4,10,15,14,9,8]$. From these comparative studies two different approaches towards movement analysis can be discerned. The first approach considers characteristics of the overall movement, such as total time or throughput (Fitts' Law). The second approach, mostly found in human movement research, assumes that a movement consists of several submovements and that this 
division may reveal distinct information about the quality of the overall movement $[10,16]$. Although both approaches have proven to be helpful in comparing input devices or interaction techniques $[15,1,9,18]$ it is difficult to use them to distinguish different movement strategies and to apply these approaches to the design of input devices and interaction techniques.

In the current paper, we first explain why we believe it is necessary to develop a new method to analyze interaction movements. Subsequently, we propose new parsing rules for decomposing an overall movement into several meaningful movement phases. To illustrate the added value of this new analysis method it is used to compare indirect mouse interactions with direct stylus interactions. It will be demonstrated that a more detailed description of how interaction movements are influenced by the input device can be obtained by dividing the movements into meaningful phases. Finally, a large number of existing and newly proposed measures were applied to assess the characteristics of the identified movement phases. The method that we propose for identifying complementary measures is another contribution of the current paper.

\section{Movement Analysis}

\subsection{Overall Movement Characteristics}

Early studies on input devices based the analysis of movements on characteristics of the overall movement. The best-known example is Fitts' law [5], which states that the time to move to a target (MT) is logarithmically related to the ratio of the distance to the target (A) over the width of the target (W), i.e.,

$$
\mathrm{MT}=\mathrm{a}+\mathrm{b} * \log _{2}(\mathrm{~A} / \mathrm{W}+1) .
$$

The logarithmic term is called the index of difficulty (ID) in "bits". It increases with a larger distance to the target or a smaller target width. The reciprocal of $b$ is the index of performance (IP) or bandwidth in "bits/s", which is one of the summary statistics that is frequently used to compare movements made under different conditions. The most popular (and standardized) summary statistic used in most recent studies is throughput (IDe/MT), where the effective width (We) of the target is used instead of the actual target width (W) in the definition of the index of difficulty $[14,17]$.

Although Fitts' law can establish that there are differences between input devices or interaction techniques, it cannot provide much insight into the factors that are responsible for these differences. MacKenzie et al. [14] acknowledged that Fitts' law measures are "gross measures" and that they lack "any information on movement during a trial". Therefore, they proposed several alternative measures, based on the path being traced during the movement, to get a more accurate measure of efficiency and to get a better idea of why some devices are more efficient than others. Although their measures reveal some of the problems experienced with input devices when pointing to a target, they still do not take into account the detailed course of the movement as a function of time, i.e., they are still characteristics of the overall movement. 


\subsection{Movement Phases}

According to Woodworth [19] rapidly aimed movements consist of an initial impulse, or ballistic phase, and a perceptually guided final control, or correction phase. The initial part of the movement is relatively fast, but as people get close to the target the movement becomes slower and is characterized by irregularities in the timedisplacement profile. In other words, the ballistic phase is programmed to reach the target and the unintended errors are corrected during the correction phase, based on sensory feedback $[16,18]$. These two phases can indeed be observed in actual displacement velocity profiles of goal-directed movements, as shown in Figure 1.

Based on this model, Meyer et al. [16] proposed parsing criteria to indicate the end of the first submovement, or ballistic phase: a) a zero-crossing of the displacement velocity from positive to negative (type 1); b) a zero-crossing of the acceleration, which is the derivative of velocity, from negative to positive (type 2); c) a zerocrossing of jerk, which is the derivative of acceleration, from positive to negative (type 3). Recent comparative studies used these criteria to divide movements into submovements in order to look at movements in more detail $[9,8,18]$. We will show that the above parsing rules often produce a subdivision into submovements that is not very meaningful. This means that the observed differences in submovement patterns obtained with this method should also be questioned.

A

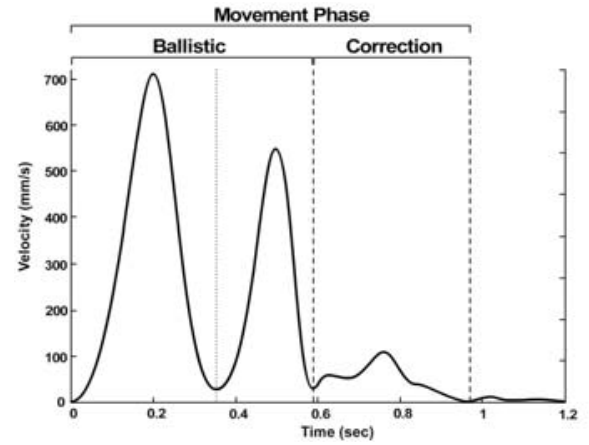

B

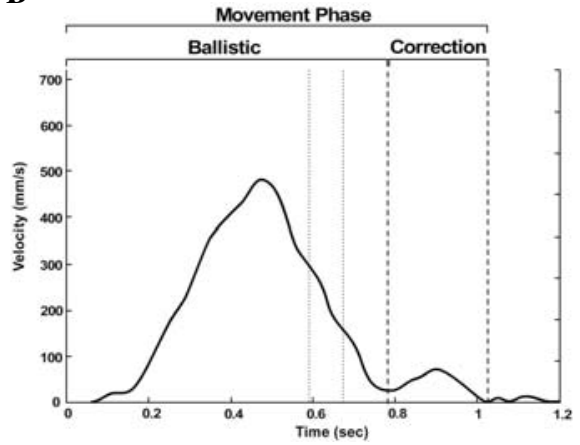

Fig. 1. Examples of velocity profiles of goal directed movements: A) executed with a mouse; B) executed with a stylus, indicating the ballistic phase and the correction phase

Although Woodworth's model formed the basis for Meyer's optimized dualsubmovement model, the criteria that Meyer et al. [16] proposed do not necessarily divide the movement into a ballistic phase and a correction phase. They assumed that goal directed movements consisted of maximally two submovements and that the ballistic phase ends after the first submovement. If one examines the velocity profiles of rapidly aimed movements, one can observe that this is frequently not the case. For example, Figure 1A shows an example of a velocity profile of a goal directed movement carried out with a mouse. This graph shows that not one but two large submovements were necessary to get into the neighborhood of the target. Another example is shown in Figure 1B, which demonstrates that when using a stylus subtle 
changes in the deceleration rate, also called type-3 submovements, can occur. According to Wisleder and Dounskaia [18] type-3 submovements occur during relatively smooth motions and are only an indication of subtle accuracy regulation. In other words, they are not believed to signal actual interruptions in the ballistic movement and, therefore, they are not likely to indicate the end of the ballistic phase.

Both graphs illustrate that the assumption that a movement maximally consists of two submovements often does not hold for two-dimensional goal directed movements. The method Meyer et al. [16] proposed was based on 1D rotation movements, for which it is more plausible that they consist of maximally two submovements. Other studies investigating 2D interactions have also demonstrated that more than two submovements occur frequently when using Meyer's criteria [9, 8, 18]. Therefore, we may conclude that the division of two-dimensional movements into submovements should be reconsidered.

\subsection{Parsing Rules}

Although Meyer's criteria allow for a more detailed analysis of movements into submovements, these criteria require adjustments to address the problems described above. We therefore propose a modified method that is more stable and reliable with respect to the differentiation between the ballistic phase and the correction phase of a movement. The method has been tested on a large number of aimed movements, executed in both 2D and 3D, with a diversity of input devices (mouse, trackball, stylus in 2D and magnetically and optically tracked stylus in 3D). The method has been proven to be more robust than the parsing rules proposed by Meyer et al. [16].

Input data filtering. We start by filtering the position data as a function of time since taking derivatives of noisy signals easily gives rise to spurious details. The data is filtered using a Gaussian time filter with a standard deviation of $25 \mathrm{~ms}$, which is comparable to the $7 \mathrm{~Hz}$ low-pass filter proposed in earlier studies. The advantage of a Gaussian filter is that it is known not to introduce spurious details, as explained in the theory of scale space filtering [12]. For this reason, Gaussian filters are used very frequently in computer vision.

Movement interval parsing. First, we identify the intervals in which actual movements occur. We define the latency phase and the verification phase as the intervals at the beginning and end of the trial where no significant movement occurs (interval in which the first and last $0.1 \mathrm{~mm}$ of the path is traveled, respectively). The latency phase can provide information about the time it takes to plan the movement towards the target. The verification phase can reveal problems with making the actual selection once the pointer has arrived at the target position (see Figure 2).

Second, the interval between the latency phase and the verification phase is divided into distinct movement intervals. These distinct movement intervals are separated by pauses in which no or only minimal movement of the pointer occurs. A pause is defined as an interval in which the speed of the pointer remains below 0.02 times the movement's peak speed. In contrast to earlier studies we propose to determine the speed of the pointer along the movement path instead of considering the parallel 
displacement of the pointer. Path length is used to determine the pointer's speed because the displacement velocity profile cannot discern real pauses from intervals in which the pointer moves perpendicular to the task axis. In addition, the analysis based on path length does not require a known task axis like the analysis based on parallel displacement, which makes it easier to extend the method to other tasks (such as circular steering

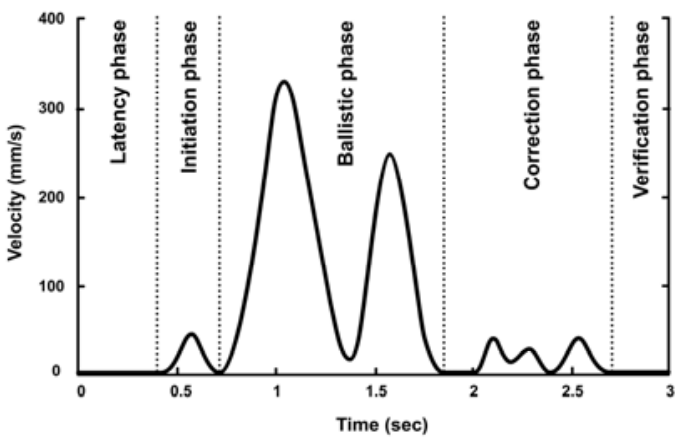

Fig. 2. Example of a velocity profile containing all 5 movement phases tasks).

Third, we determine for each identified movement interval whether or not it makes a considerable contribution to approaching the target. If the path length of a movement interval is contributing more than $25 \%$ to the total path length it is considered to be part of the ballistic phase. This criterion is introduced to be able to deal with cases where several movements are required to reach the target, such as the one depicted in Figure 1A. If a movement interval does not make a considerable contribution it is considered to be part of either the correction phase or the initiation phase. The movement interval will be considered part of the correction phase when it occurs after the last ballistic movement and it will be considered part of the initiation phase when it occurs before the first ballistic movement.

Finally, we divide the separate movement intervals into submovements. We do this for two reasons. First, we use this division to get more detailed information on how the movement was performed (i.e., fluently or with corrections). Second, we use this division to determine whether or not the last movement interval of the ballistic phase contains some correction submovements at the end. The criteria proposed by Meyer et al. [16] were adjusted so they could be applied to speed profiles based on path length:

a) a type-1 submovement occurs when the speed becomes (almost) zero (less than 0.02 times the movement's peak speed, which only occurs at the beginning of a movement interval);

b) a type- 2 submovement occurs at a zero-crossing of acceleration from negative to positive (in combination with a positive jerk that exceeds 0.01 times the maximally observed jerk);

c) a type-3 submovement occurs at a zero-crossing of jerk from positive to negative (in combination with a negative value of its derivative that exceeds 0.01 times the maximally observed value).

The thresholds on the slopes of the zero crossings are incorporated to avoid submovement detection during small involuntary tremor or slow drift. The minimal requirements for submovements that were proposed by Meyer et al. [16] were specific for their 1D rotation task, and needed to be adapted for the case of 2D movement path. The following minimal requirements for a submovement (to avoid the division of movements into meaningless small submovements) are: a submovement should 
traverse a distance of at least $2 \mathrm{~mm}$ and last for at least $75 \mathrm{~ms}$, while the maximum velocity should exceed 0.02 times the maximally observed velocity. Submovements that do not meet this requirement are combined with bordering submovements.

If the last movement in the ballistic phase consists of multiple submovements the ballistic phase ends at the first type- 2 submovement that occurs in the last $75 \%-95 \%$ of the traveled path length. The corrective submovements that occur during the final part of the interaction movement are considered to assist in positioning the pointer within the target boundaries. They should hence be considered as being part of the correction phase. As mentioned before, type-3 submovements are only considered to be indications of subtle accuracy regulation and are therefore not used to signal the end of the ballistic phase. If the last ballistic movement consists of only one submovement the end of this movement coincides with the end of the ballistic phase.

\subsection{Performance Measures}

In order to draw meaningful conclusions from interaction movements a division of movements into meaningful components is not sufficient. We also need summary measures to assess the key characteristics of the phases that have been distinguished. A review of 43 studies in the field of $2 \mathrm{D}$ interaction and human movement science resulted in a set of 63 measures that can be applied to evaluate $2 \mathrm{D}$ interaction techniques. These findings indicate that there is no consensus about which measures are best to use when evaluating input devices and interaction techniques. It is highly likely, however, that several proposed measures assess similar qualities of the movement. It is argued that from this large list of measures only a few measures should be retained, i.e. those that are complementary to each other.

In the current study only measures that can extract information from a single movement path were applied to the movement data. We made an exception for throughput, which extracts the effective width from a series of trials in the same condition, for it is included in the ISO standard (ISO 9241-9). Time is one of the most frequently used measures to assess movement quality and therefore it was applied to the total trial and to each of the movement phases (latency, initiation, ballistic, correction and verification phase). Besides time and phase occurrence, 47 measures were applied to the overall movement, the ballistic phase and/or the correction phase. A short description of these measures can be found in Appendix 1. As indicated in the appendix some of the measures were applied to the overall movement as well as to the ballistic phase and the correction phase and some of the measures were applied to only the overall movement, the ballistic phase or the correction phase. As a result, a total of 99 movement characteristics were obtained from the data.

As mentioned before, the study described in the current paper compares indirect mouse interactions with direct stylus interactions. The reason for this choice is that previous studies comparing mouse interactions with stylus and tablet interactions were only able to find minor differences between the mouse and the stylus $[15,1]$, especially with respect to movement time. We will show how the application of the new division method in combination with the use of alternative measures can indeed provide a more detailed description of indirect mouse interaction movements and direct stylus interaction movements. 


\section{Experiment}

\subsection{Participants}

Eight university employees voluntarily participated in the study that we undertook to test the proposed measures. The group consisted of 5 males and 3 females. Their age ranged from 30 to 35 years $(M=32.4$ years $)$. All participants indicated that their right hand was their preferred hand when using the mouse. Two participants indicated that their left hand was the preferred hand when using the stylus.

\subsection{Task}

The task used in the experiment was a multi-directional pointing task. In this task 8 "target" circles are arranged in larger circles with a diameter of 48, 96 and $144 \mathrm{~mm}$ around a central "home" circle (see Figure 3A). The targets have three different sizes, $3 \mathrm{~mm}, 6 \mathrm{~mm}$ and $9 \mathrm{~mm}$. The 9 combinations of target size and target distance resulted in 7 different levels of difficulty (ID $=2.7,3.2,3.5,4.1,4.6,5$ and 5.6 bits). At the beginning of a new trial, the target was presented together with the home circle (3 $\mathrm{mm})$. The targets were presented in random order, with the restriction that subsequent targets were never positioned in the same direction in order to prevent learning effects. The data collection started when the home-circle was selected and continued until the target was correctly selected.

A

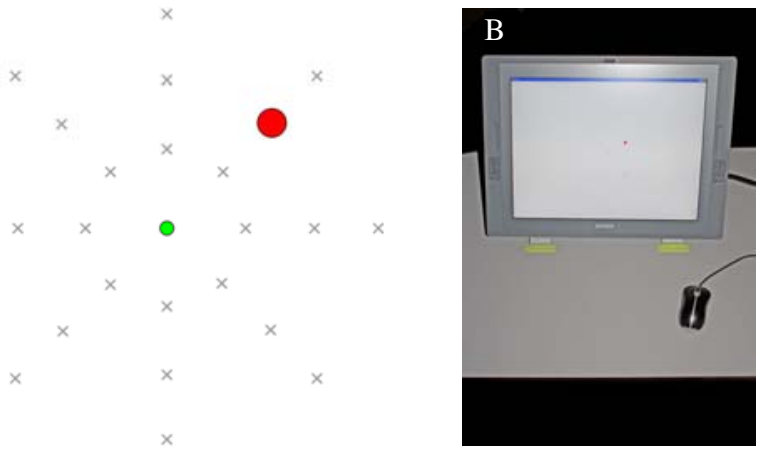

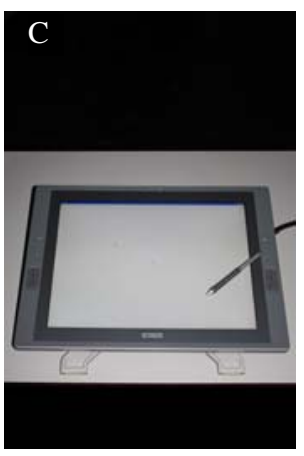

Fig. 3. Multi-directional pointing task: A) target layout, indicating home-circle (middle), targetcircle and the other possible target positions; B) screen set-up when using mouse; C) screen setup when using stylus

\subsection{Procedure}

The selection task was presented on a 21-inch WACOM Cintiq 21UX tablet, with integrated display. The resolution of the screen was set at 1600x1200 pixels. The position of the screen was changed in between sessions: when participants were using the mouse the screen was positioned vertically (see Figure 3B) and when they were using the stylus the screen was tilted horizontally so that the screen would face up (see Figure 3C). The position of the screen was adjusted to simulate the normal way 
of use as much as possible. The mouse had a constant CD-ratio of 1:4, while the CDratio was 1:1 in case of the stylus with integrated display.

Before participants started the experiment they were provided with a short instruction about the task. The experiment consisted of two sessions, each containing 72 trials, i.e., 8 target directions combined with 3 different target sizes and 3 different target distances. A practice session with 27 trials, i.e., 3 target directions combined with 3 different target sizes and 3 different target distances, preceded each actual experimental session. The order of the two experimental sessions was balanced so that the number of participants using the mouse during the first session was equal to the number of participants using the stylus during the first session.

\section{Results}

Although target size and target distance were included in the statistical model, the analysis reported here will focus on the influence of input device on phase duration and phase occurrence. Before analyses were carried out log transformations were applied to time measures and square root transformations were applied to counts. These transformations accomplished that the distributions of the data were closer to the normal distribution assumed by the applied statistical methods (such as ANOVA).

\subsection{Overall Movement vs. Movement Phases}

Figure 4A shows the duration of the total trial, ballistic phase and correction phase as a function of the input device. We averaged the times for the 8 different directions and applied a within-subject correction for subject, target size and target distance as proposed by Loftus and Masson [13]. As can be seen from this figure there is no difference in total trial duration between the mouse and the stylus. However, it also shows that there are considerable differences in the duration of the ballistic phase, correction phase and verification phases between the mouse and the stylus.

The repeated measures analysis with target size $(3 \mathrm{~mm} / 6 \mathrm{~mm} / 9 \mathrm{~mm})$ and target distance $(48 \mathrm{~mm} / 96 \mathrm{~mm} / 144 \mathrm{~mm})$ as within-subjects variables confirmed that there was no significant difference in total trial time as a function of input device (mouse/stylus), $F(1,7)=.08, p=.79$. It also confirmed that there were significant differences in duration of the ballistic phase and the correction phase between the mouse and the stylus. The ballistic phase was shorter when participants were using the mouse $(M=.41 ; S E=.02)$ than when they were using the stylus $(\mathrm{M}=.64 ; S E=.02), F(1,7)=137.73, p<.01$. On the other hand, the correction phase was longer when participants were using the mouse $(M=.34 ; S E=.01)$ than when they were using the stylus $(M=.23 ; S E=.01)$, $F(1,7)=72.38, p<.01$.

Repeated measures were also carried out to test the effect of input device on the duration of the latency phase, start phase and verification phase. Only the duration of the verification phase showed a significant effect of input device. The verification phase was longer when using the mouse $(M=.18 ; S E=.01)$ than when using the stylus $(M=.07 ; S E=.01), F(1,7)=97.16, p<.01$. Due to the infrequent occurrence of the initiation phase when participants were using the mouse (see Figure 4B) the repeated measures analysis could not be carried out on the duration of the initiation phase. 

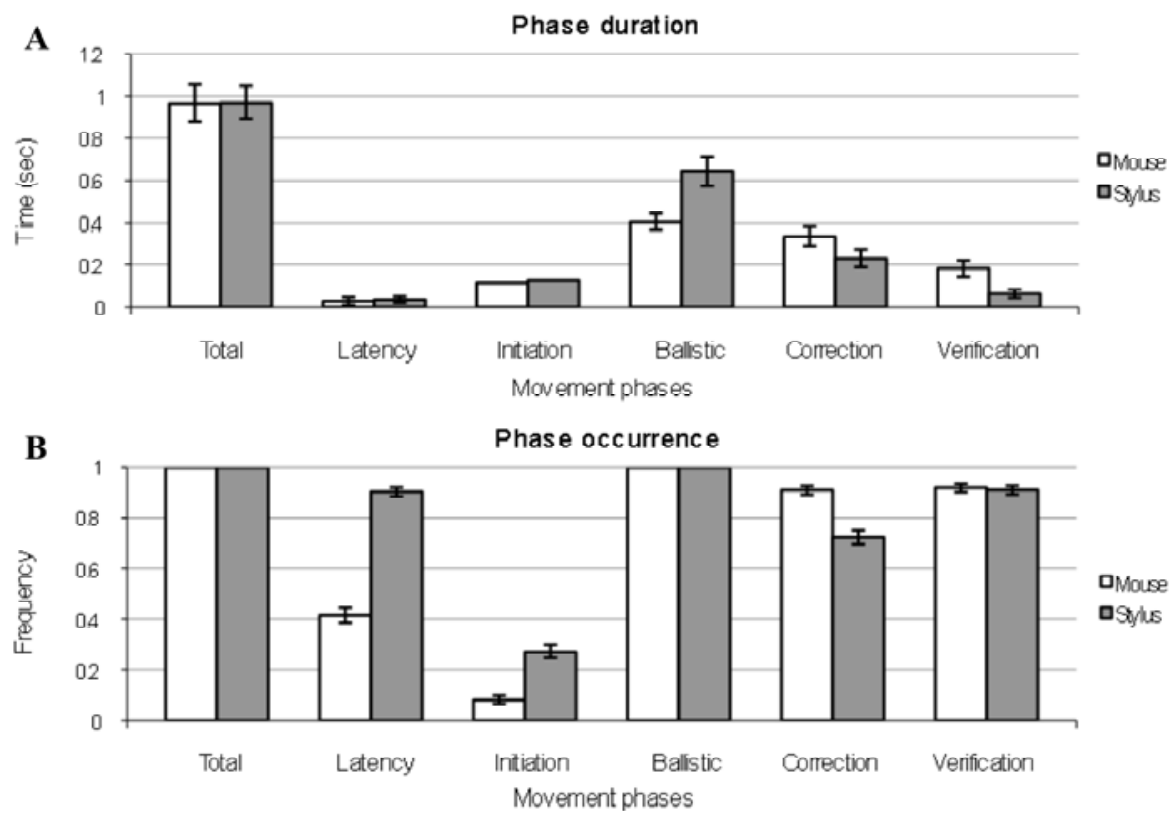

Fig. 4. The duration and phase occurrence (with $95 \%$ confidence intervals) are shown as a function of input device (mouse/stylus) for each movement phase

Figure 4B shows the frequency of occurrence for each of the movement phases as a function of the input device. This figure clearly illustrates that the latency phase and the initiation phase occur more often when participants were using the stylus than when they were using the mouse. However, the correction phase occurs more frequently when participants were using the mouse.

\subsection{Measure Selection}

To select only a few measures to assess the quality of the interaction movements a principal components factor analysis with varimax rotation was carried out. Fourteen of the 99 movement characteristics (such as initiation time, number of pauses during the ballistic phase, number of target misses and number of slip-offs) were excluded from the factor analysis because the data distributions were strongly skewed. By default, factor analysis retains factors with an eigenvalue larger than 1. However, this method resulted in the extraction of 15 factors, which would imply a relatively large selection of measures. Therefore, we chose to increase the eigenvalue boundary to 4 , which resulted in the extraction of 4 factors, with eigenvalues equal to 22.4 , 19.0, 8.0 and 4.9. Together, these factors explain almost $65 \%$ of the variance, with the last factor still making a considerable contribution to the explained variance $(5.8 \%)$. 
Table 1. Factor loadings after varimax rotation on the 4 extracted factors

\begin{tabular}{|c|c|c|c|}
\hline Factor 1 & Factor 2 & Factor 3 & Factor 4 \\
\hline $\begin{array}{l}\text { Duration ballistic } \\
\text { phase }\end{array}$ & $\begin{array}{l}\text { Peak speed ballistic } \\
\text { phase }\end{array}$ & $\begin{array}{l}\text { Pauses }>100 \mathrm{~ms} \\
\text { correction phase }\end{array}$ & $\begin{array}{l}\text { Orthogonal } \\
\text { direction change } \\
\text { overall movement }\end{array}$ \\
\hline $\begin{array}{l}\text { Mouse } M=.41 ; S E=.02 \\
\text { Stylus } M=.64 ; S E=.02 \\
F(1,7)=137.73 ; p<.01\end{array}$ & $\begin{array}{l}\text { Mouse } M=547.3 ; \text { SE }=17.2 \\
\text { Stylus } M=334.4 \text { SE }=26.9 \\
F(1,7)=57.95 ; p<.01\end{array}$ & $\begin{array}{l}\text { Mouse } M=.29 ; S E=.02 \\
\text { Stylus } M=.32 S E=.03 \\
F(1,7)=.26 ; p=.63\end{array}$ & $\begin{array}{l}\text { Mouse } M=.66 ; S E=.05 \\
\text { Stylus } M=.97 ; S E=.10 \\
F(1,7)=2.34 ; p=.17\end{array}$ \\
\hline $\begin{array}{l}\text { Relative accuracy } \\
\text { ballistic phase }\end{array}$ & $\begin{array}{l}\text { Path length overall } \\
\text { movement }\end{array}$ & $\begin{array}{l}\text { Duration } \\
\text { correction phase }\end{array}$ & $\begin{array}{l}\text { Movement offset } \\
\text { overall movement }\end{array}$ \\
\hline $\begin{array}{l}\text { Mouse } M=.12 ; S E=.005 \\
\text { Stylus } M=.05 ; S E=.003 \\
F(1,7)=251.60 ; p<\end{array}$ & $\begin{array}{l}\text { Mouse } M=107.4 ; S E=2.8 \\
\text { Stylus } M=101.6 ; S E=1.9 \\
F(1,7)=5.83, p<.05\end{array}$ & $\begin{array}{l}\text { Mouse } M=.34 ; S E=.01 \\
\text { Stylus } M=.23 ; S E=.01 \\
F(1,7)=72.38 ; p<.01\end{array}$ & $\begin{array}{l}\text { Mouse } M=3.0 ; S E=.18 \\
\text { Stylus } M=2.8 ; S E=.64 \\
F(1,7)=.14 ; p=.72\end{array}$ \\
\hline $\begin{array}{l}\text { Number of type } 3 \\
\text { submovements } \\
\text { ballistic phase }\end{array}$ & $\begin{array}{l}\text { Mean speed ballistic } \\
\text { phase }\end{array}$ & $\begin{array}{l}\text { Mean duration } \\
\text { of pauses } \\
\text { correction phase }\end{array}$ & $\begin{array}{l}\text { Path length } \\
\text { efficiency ballistic } \quad-.60 \\
\text { phase }\end{array}$ \\
\hline $\begin{array}{l}\text { Mouse } M=.08 ; S E=.01 \\
\text { Stylus } M=.54 ; S E=.04 \\
F(1,7)=30.47 ; p<.01\end{array}$ & $\begin{array}{l}\text { Mouse } M=234.8 ; S E=11.0 \\
\text { Stylus } M=146.0 ; S E=7.7 \\
F(1,7)=75.31 ; p<.01\end{array}$ & $\begin{array}{l}\text { Mouse } M=.15 ; S E=.01 \\
\text { Stylus } M=.13 ; S E=.01 \\
F(1,7)=10.25 ; p<.05\end{array}$ & $\begin{array}{l}\text { Mouse } M=.99 ; S E=.05 \\
\text { Stylus } M=.98 ; S E=.09 \\
F(1,7)=16.81 ; p<.01\end{array}$ \\
\hline
\end{tabular}

As can be observed in Table 1, the groups of measures loading high on the 4 resulting factors focus on different aspects of the goal directed movement. The first factor mostly contains measures that assess features of the ballistic phase and how it is related to the overall movement. The second factor focuses on how the movement is executed in terms of the length of the traveled path and speed. The third factor typically contains measures that assess the quality of the correction phase, e.g. the number and duration of pauses. The final factor includes measures that mainly focus on the efficiency of the movement path, like the number of orthogonal direction changes and the movement offset. This fourth factor hence resembles the measures proposed by MacKenzie et al. [14].

Table 1 also shows the results of repeated measures analyses which were carried out to test the main effect of input device. The results show that the duration of the ballistic phase was longer when participants were using the stylus, mainly because they were moving slower. Although more online adjustments were made during the ballistic movement and the ballistic phase ended closer to the target, the path length efficiency of the ballistic phase was somewhat lower when participants were using the stylus. When looking at the correction phase, there is no difference between input devices with respect to the number of pauses. However the duration of pauses is somewhat longer when participants use the mouse. Finally, there are also no differences between the input devices with respect to the number of orthogonal direction changes and the movement offset.

The measures extracted by the factor analysis were able to provide a well-founded explanation for the differences found in the duration of the distinct movement phases. The measures that proved useful in the analysis besides movement time and phase 
occurrence (frequency) are therefore proposed to assess the characteristics of aimed movements, especially with respect to differentiating between input devices:

Mean speed
Peak speed
Path length
Path efficiency
Relative accuracy ballistic phase
Orthogonal direction change

Movement offset

Number of submovements (type 3)

Number and duration of pauses

Most of the proposed measures can be applied to the total trial as well as the ballistic phase and the correction phase, like mean speed, path length and movement offset. This means that these measures are also able to specify differences between these phases.

\section{Conclusion and Discussion}

This paper has introduced parsing criteria that divide a goal-directed movement not only into a ballistic and a correction phase, but also into an initiation phase, latency phase and verification phase. Although the criteria we proposed deviate from Meyer's criteria and might seem arbitrary it is not our primary aim to provide criteria that can be theoretically founded. It is our aim to provide a method that is able to divide goaldirected movements into meaningful parts. We believe that the division into the five phases described above is a meaningful and intuitive division. In addition, since the analysis method is applied to the cursor position this analysis method of goal directed movements can also be applied to assess the performance of other input device or interaction techniques in 2D as well as 3D environments.

We have demonstrated that the analysis of rapidly aimed movements into five movement phases (latency phase, initiation phase, ballistic phase, correction phase and verification phase) provides insights that cannot be obtained from the analysis of the overall movement. Due to the reverse effects of the input devices on the duration of the different phases, no main effects for input device can be found when looking at the overall movement duration. It is expected that such effects do not only occur with respect to movement time but also with respect to other measures. Even with an observed difference in the overall movement it is very well possible that the effect can be solely attributed to a particular phase. For example, the measure number of pauses reveals significant differences between the mouse and the stylus when looking at the overall movement. However, pauses are mainly found in the correction phase and not in the ballistic phase. Therefore, the application of a range of measures to the separate movement phases enables us to better understand the root causes of the differences between input devices. In this way the proposed analysis method is especially helpful in the design and improvement of input devices and interaction techniques.

The other contribution of the current paper is that we reduced the list of measures that can be applied to goal-directed movements to just a few measures. This selection 
was carried out because some measures assess similar aspects of the movements and are therefore redundant and some measures are better in discriminating between variables than others. It is thought that the selected measures have some face validity and they assisted very much in explaining the differences between mouse and stylus interactions. For example, from the selected measures it can be concluded that, when using the stylus, the ballistic movement is considerably slower and more online adjustments are made. As a result, the execution is more precise and, therefore, less time is required to enter the target region, which is shown by the shorter correction phase. A different strategy is applied when participants are using the mouse. Not only the short duration of the ballistic phase but also the absence of the latency phase and initiation phase indicate that participants using the mouse want to get into the neighborhood of the target as fast as possible. However, since the movement is fast and less controlled more time is required to make the final corrections in order to enter the target area.

Although the precise outcome may somewhat depend on the difference in CD-ratio for the mouse and stylus, it still shows areas where the mouse and stylus interactions can be improved. For the improvement of the mouse interactions, the focus should lie on increasing the end-point accuracy of the ballistic movement and on facilitating the execution of the correction movements, for example by automation techniques. On the other hand, for the improvement of the stylus interactions more focus should lie on increasing the movement speed, while maintaining the movement accuracy. Another issue with respect to direct stylus interactions is that participants sometimes covered the targets with their arm and as a result started moving in the wrong direction. This is also shown by a lower path length efficiency when participants were using the stylus. Solving the problem of covering important content would also increase performance of direct stylus interactions.

The proposed analysis method is tailored to assess the quality of rapidly aimed selection movements. Steering is another interaction technique that is frequently used in computer interactions (e.g. navigation through menu-structures). A major difference between selection and steering tasks is that steering tasks require a more continuous control of pointer precision. As a result, steering movements will most likely be subject to ongoing corrections and might have different characteristics than goal directed movements. Future work will focus on the question how the analysis method can be adjusted so that it can also be applied to these more controlled movements. Besides selection and navigating though menu-structures, there are other tasks that are important with respect to computer interactions such as changing an object's position and/or its orientation (e.g. in graphical applications). These tasks have not been standardized yet and future work should also focus on designing simple tasks that have similar characteristics as the ISO 9241-9 pointing task. In order to investigate the performance on these different tasks in a systematic way, it should also be investigated whether similar factors and measures will be extracted when different interaction techniques or tasks are applied.

\section{Acknowledgments}

This research was supported by The Netherlands Organization for Scientific Research (NWO). We would like to thank Victor Vloemans for the technical assistance. 


\section{References}

1. Accot, J., Zhai, S.: Performance Evaluation of Input Devices in Trajectory-Based Tasks: An Application of the Steering Law. In: Proceedings of CHI 1999, pp. 466-472 (1999)

2. Akamastu, M., MacKenzie, I.S., Hasbrouq, T.: A Comparison of Tactile, Auditory, and Visual Feedback in a Pointing Task Using a Mouse-Type Device. Ergonomics 38, 816827 (1995)

3. Behbehani, K., Kondraske, G.V., Richmond, J.R.: Investigation of Upper Extremity Visuomotor Control Performance Measures. IEEE Transactions on Biomedical Engineering 35, 518-525 (1988)

4. Card, S.K., English, W.K., Burr, B.J.: Evaluation of Mouse, Rate-Controlled Isometric Joystick, Step Keys, and Text Keys for Text Selection on a CRT. Ergonomics 21, 601-613 (1978)

5. Fitts, P.M.: The Information Capacity of the Human Motor System in Controlling the Amplitude of Movement. Journal of Experimental Psychology 47, 381-391 (1954)

6. Goldvasser, D., McGibbon, C.A., Krebs, D.E.: High Curvature and Jerk Analyses of Arm Ataxia. Biological Cybernetics 84, 85-90 (2001)

7. Hansen, S., Tremblay, L., Elliott, D.: Real-Time Manipulation of Visual Displacement during manual aiming. Human Movement Science 27, 1-11 (2008)

8. Hourcade, J.P.: Learning from Preschool Children's Pointing Submovements. In: Proceedings of IDC 2006, pp. 65-72 (2006)

9. Hwang, F., Keates, S., Langdon, P., Clarkson, J.: A Submovement Analysis of Pointer Trajectories. Behaviour and Information Technology 24, 205-217 (2005)

10. Jagacinski, R.J., Repperger, D.W., Moran, M.S., Ward, S.L., Glass, B.: Fitts' Law and the Microstructure of Rapid Discrete Movements. Journal of Experimental Psychology: $\mathrm{Hu}-$ man Perception and Performance 6, 309-320 (1980)

11. Keates, S., Trewin, S.: Effect of Age and Parkinson's Disease on Cursor Positioning Using a Mouse. In: Proceedings of ASSETS 2005, pp. 68-75 (2005)

12. Koenderink, J.J.: The Structure of Images. Biological Cybernetics 50, 363-370 (1984)

13. Loftus, G.R., Masson, M.E.J.: Using Confidence Intervals in Within-Subject Designs. Psychonomic Bulletin \& Review 4, 476-490 (1994)

14. MacKenzie, I.S., Kauppinen, T., Silfverberg, M.: Accuracy Measures for Evaluating Computer Pointing Devices. In: Proceedings of CHI 2001, pp. 9-16 (2001)

15. MacKenzie, I.S., Sellen, A., Buxton, W.A.: Comparison of Input Devices in Elemental Pointing and Dragging Tasks. In: Proceedings of CHI 1991, pp. 161-166 (1991)

16. Meyer, D.E., Abrams, R.A., Kornblum, S., Wright, J.E., Smith, J.E.K.: Optimality in Human Motor Performance: Ideal Control of Rapid Aimed Movements. Psychological Review 95, 340-370 (1988)

17. Soukoreff, R.W., MacKenzie, I.S.: Towards a Standard for Pointing Device Evaluation, Perspective on 27 Years of Fitts' Law Research in HCI. International Journal of HumanComputer Studies 61, 751-789 (2004)

18. Wisleder, D., Dounskaia, N.: The Role of Different Submovement Types During Pointing to a Target. Experimental Brain Research 176, 132-149 (2007)

19. Woodworth, R.S.: Accuracy of voluntary movement. Psychological Review, Monograph Supplement 3, 1-114 (1899) 


\section{Appendix 1. Measures with Descriptions}

Acceleration time: Time interval during which the pointer was accelerating' [18]

Angle offset: Angle between the vector from the beginning of the ballistic phase to the end of the ballistic phase and the task axis ${ }^{2}[8]$

Average speed: Average speed in $\mathrm{mm} / \mathrm{sec}$, i.e. total path length divided by total time ${ }^{1}$

Counter-productive submovements: Number of times the coincident error is negative with a magnitude larger than the submovement's starting distance ${ }^{1}[9]$

Deceleration time: Time interval during which the pointer was decelerating ${ }^{1}[18]$

Distance to peak speed: Distance traveled from movement onset to the moment peak speed is reached ${ }^{2}$

Error magnitude: Distance from the position of the target miss (button click) to the target edge

Final positioning time: Interval from target entry until the end of the $\operatorname{trial}^{3}[2]$

Goal distance correction phase: Distance to the target at the start of the correction phase ${ }^{3}$

High curvature occurrence: Number of times the angle between 3 sample points is less than $80 \operatorname{deg}^{1}$ [6]

Length offset: Difference between the length of the ballistic phase and 'distance to target' at the beginning of the ballistic phase ${ }^{2}[8]$

Max percent overshoot: largest percent deviation from the target once the pointer passes the $\operatorname{target}^{2}$ [3]

Movement direction change: Number of times the tangent to the path gets parallel to the task axis ${ }^{1}[14]$

Movement error: Mean of absolute distances of the path from the task axis ${ }^{1}$ [14]

Movement offset: Overall mean distances of the path from the task axis ${ }^{1}[14]$

Movement time: time interval from movement onset to movement offset ${ }^{4}[16]$

Movement variability: Extent to which the path lies in a straight line parallel to the task axis ${ }^{1}$ [14]

Orthogonal direction change: Number of times the tangent to the path becomes perpendicular to the task $\operatorname{axis}^{1}[14]$

Overshoot: the frequency and duration (time interval from the moment the pointer passes the target edge to movement offset) of overshoots ${ }^{2}[9,3]$

Path length: Length of the path in $\mathrm{mm}^{1}$

Path length efficiency: Ratio between the shortest path and the traveled path ${ }^{1}[11]$

Pauses: Number of pauses (>0ms; $>100 \mathrm{~ms}$; $>250 \mathrm{~ms}$ ) [11] and mean duration of the pauses ${ }^{1}[9]$

Peak acceleration: Maximum acceleration reached during the overall movement ${ }^{2}[7]$

Peak deceleration: Maximum deceleration reached during the overall movement ${ }^{2}[7]$

Peak speed: Maximum speed reached during the movement ${ }^{2}$ [11]

Peak time: time at which maximum overshoot is reached ${ }^{2}[3]$

Perpendicular error: Distance between the endpoint of the ballistic phase and the task axis, measured in the direction normal to the task axis ${ }^{2}$ [9]

Relative accuracy: Ratio between 'distance to target' at the end of the ballistic phase and at movement onset $^{2}[10]$

Relative parallel displacement: Ratio between parallel displacement of the ballistic phase and the overall movement ${ }^{2}$

Relative path length: Ratio between path length of the ballistic phase and the overall movement ${ }^{2}$ [11]

Relative time: Ratio between duration of the ballistic phase and the overall movement ${ }^{2}[18]$

Relative time to peak speed: Ratio between time from movement onset to peak speed and the overall movement ${ }^{2}[11]$

Slip-off: Number of submovements that begin inside the target but end outside the $\operatorname{target}^{3}$ [9]

Submovements: Number of submovements [9] and number of type 1, 2 and 3 submovements ${ }^{1}$ [18]

Submovements after target entry: Number of additional submovements after the pointer has landed inside the target until movement offset ${ }^{3}$ [9]

Target miss: Frequency of trials in which the mouse button is clicked outside the target $\operatorname{area}^{3}[9]$

Target re-entry: Number of times the pointer enters the target after the first target entry ${ }^{3}$ [14]

Task axis crossing: Number of times the path crosses task axis ${ }^{1}[14]$

Throughput: IDe/MT $\left(\mathrm{IDe}=\log _{2}[\mathrm{D} / \mathrm{We}+1] \text {, where } \mathrm{We}=4.133 * \sigma\right)^{4}[17]$

Time to peak speed: Time interval from movement onset to the moment the peak speed is reached ${ }^{2}[11]$

1 Applied to the overall movement, the ballistic phase and the correction phase.

2 Applied to only the ballistic phase.

3 Applied to only the correction phase.

${ }^{4}$ Applied to only the overall movement. 